\title{
Altered Levels of Serum Ceramide, Sphingosine and Sphingomyelin Are Associated with Colorectal Cancer: A Retrospective Pilot Study
}

\author{
DUSKA SEPAROVIC ${ }^{1,2}$, ANTHONY F. SHIELDS ${ }^{2,3}$, PHILIP A. PHILIP ${ }^{2,3}$, \\ JACEK BIELAWSKI ${ }^{4}$, ALICJA BIELAWSKA ${ }^{4}$, JASON S. PIERCE ${ }^{4}$ and ADI L. TARCA ${ }^{5,6}$ \\ ${ }^{1}$ Department of Pharmaceutical Sciences, Eugene Applebaum College of Pharmacy and Health Sciences, \\ ${ }^{2}$ Karmanos Cancer Institute, Departments of ${ }^{3}$ Oncology and ${ }^{5}$ Obstetrics and Gynecology, School of Medicine, and \\ ${ }^{6}$ Department of Computer Science, College of Engineering, Wayne State University, Detroit, MI, U.S.A.; \\ ${ }^{4}$ Department of Biochemistry and Molecular Biology, Medical University of South Carolina, Charleston, SC, U.S.A.
}

\begin{abstract}
Background/Aim: Because patients with cancer of apparently equivalent stage often have different outcomes, it is necessary to gather additional information to complement cancer staging. Dysregulated sphingolipid metabolism contributes to carcinogenesis. In this retrospective pilot study, we tested the hypothesis that changes in serum levels of sphingolipids are associated with stage IV colorectal cancer $(C R C)$. Patients and Methods: We used commercially available serum samples from healthy males and patients with CRC (adenocarcinoma of the large intestine, stage IV with metastases). Blood samples were obtained immediately prior to anesthesia/surgery. We measured sphingolipid levels in sera using mass spectrometry. Results: In serum of patients with CRC, the levels of C16-, C18-, C18:1-, and C24:1-ceramide, as well as those of sphingosine, were significantly higher than those of controls. In contrast, the levels of C24-sphingomyelin were significantly lower than those of controls. A global test of association showed that ceramides and sphingomyelins but not hexosylceramides were significantly associated with stage IV CRC. Conclusion: Sphingolipids have a potential of serving as novel, non-invasive, inexpensive, and effective blood-based biomarkers to complement CRC staging for better prognosis and more personalized medicine.
\end{abstract}

This article is freely accessible online.

Correspondence to: Dr. Duska Separovic, Department of Pharmaceutical Sciences, Eugene Applebaum College of Pharmacy and Health Sciences, Wayne State University, 259 Mack Ave., Detroit, MI 48201, U.S.A. Tel: +1 3135778065, Fax: +1 3135772033, e-mail: jmjilyss999@gmail.com.

Key Words: Biomarkers, ceramide, colorectal cancer, serum, sphingolipids.
Colorectal cancer (CRC) is the third most commonly diagnosed cancer and the third leading cause of cancerrelated deaths in the United States. The American Cancer Society estimated that 95,270 new cases of colon cancer and 39,220 new cases of rectal cancer would occur in the United States in 2016. CRC was expected to cause about 49,190 deaths in 2016 (http://www.cancer.org/acs/groups/content). Current staging methods for cancer, including CRC, provide only probable outcomes of the disease course, with considerable differences in outcomes for patients with cancer of apparently equivalent stage. Thus, in order to provide patients with a more accurate prognosis, there is a need to develop additional biomarkers that can be used to complement cancer stratification systems (1). Bioactive sphingolipids, whose dysregulated metabolism is associated with CRC (2), represent potential noninvasive blood-based biomarkers that could meet this need.

Sphingolipid metabolism includes ceramide generation from sphingomyelin via sphingomyelinase (SMase) and via the de novo sphingolipid biosynthesis pathway. This pathway includes a ceramide synthase (CerS)-dependent acylation step, in which a fatty acyl group is added to dihydrosphingosine to form dihydroceramide (Figure 1). Ceramide is formed from dihydroceramide via desaturasedependent reaction. The members of the ceramide and dihydroceramide families differ depending on the fatty acyl chain they contain. CerS1 and CerS6 are involved in the production of $\mathrm{C} 18$ - and $\mathrm{C} 16$-ceramide, containing the 18and 16-fatty acyl chains, respectively. Ceramide can be incorporated into glucosylceramide via glucosylceramide synthase or converted to sphingosine via ceramidase.

Dysregulation of sphingomyelinase and glucosylceramide synthase have been shown in tumors of patients with CRC (3, 4). Up-regulation of ceramide synthases, including CerS1 and CerS6, has been shown in cancer types other than CRC $(5,6)$. 


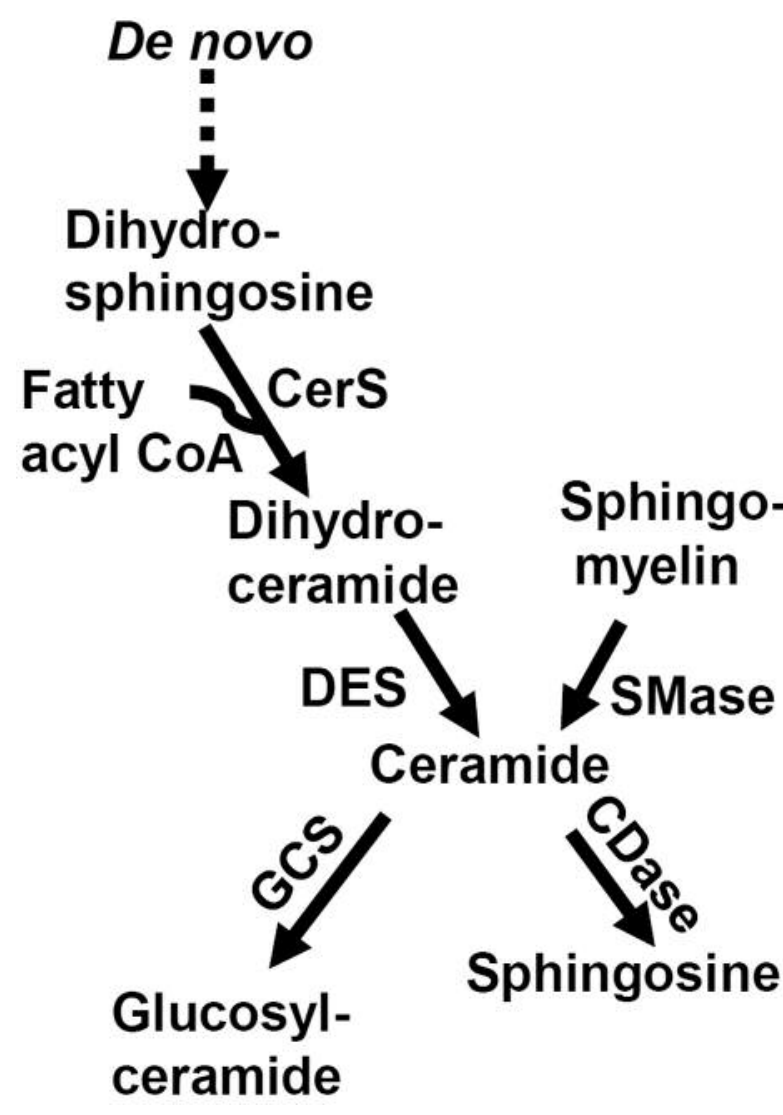

Figure 1. Sphingolipid metabolism. CDase, Ceramidase; CerS, ceramide synthase; DES, desaturase; GCS, glucosylceramide synthase; SMase, sphingomyelinase.
It is not known, however, whether blood sphingolipid levels are indicative of CRC stage. Specifically, the objective of this retrospective pilot study was to determine whether serum levels of sphingolipids are associated with stage IV CRC.

\section{Patients and Methods}

Participants and samples. Patients with stage IV CRC $(\mathrm{n}=10)$ and normal donors $(\mathrm{n}=10)$ were all Caucasian males. For normal donors, the average age was 56 (range $=44-61$ ) years. For patients with CRC, the average age was 62 (range $=44-80$ ) years; all had adenocarcinoma of the large intestine, stage IV with metastases. The details of staging and classification for the patients with CRC is provided in Table I. Each classification type was represented by one patient.

The sera of all participants were purchased from Asterand (Detroit, MI, USA). Because serum collection procedures affect normal serum sphingolipid levels in healthy individuals (7), in order to obtain reliable measurements of serum sphingolipid levels, a standardized protocol for serum collection, freezing, and shipping is required. A global network of clinical sites that provide Asterand with biosamples is expected to comply fully with those standards, as well as conduct subject recruitment, obtain informed consent, and conduct scientific activities in accordance with applicable laws, regulations, and ordinances (cf. http://www.asterandbio.com/ company/ethics/). At a clinical site, after initial diagnosis, blood was drawn immediately prior to anesthesia/surgery. The frozen sera were shipped on dry ice from Asterand to the Lipidomics Core (Medical University of South Carolina, Charleston, SC, USA) for mass spectrometric analysis.

Mass spectrometric analysis of sphingolipids. After thawing, lipids were extracted from 100- $\mu$ l aliquots of sera. Sphingolipids were separated by high-performance liquid chromatography, introduced

Table I. Staging and classification of patients with CRC according to National Comprehensive Cancer Network Clinical Practice Guidelines in Oncology: Colon Cancer (emedicine.medscape.com). Each classification type was represented by one patient.

Stage Classification

IV T2N2bM1: Tumor invades muscularis propria; metastasis in 7 or more regional lymph nodes; distant metastasis.

T3N0M1: Tumor invades through the muscularis propria into the pericolorectal tissues; no regional lymph node metastasis; distant metastasis.

T3N1bM1: Tumor invades through the muscularis propria into the pericolorectal tissues; metastasis in 2-3 regional lymph nodes; distant metastasis.

IVA T3N0M1a: Tumor invades through the muscularis propria into the pericolorectal tissues; no regional lymph node metastasis; metastasis confined to 1 organ or site (e.g. liver, lung, ovary, nonregional node).

T3N1bM1a: Tumor invades through the muscularis propria into the pericolorectal tissues; metastasis in 2-3 regional lymph nodes; metastasis confined to 1 organ or site (e.g. liver, lung, ovary, nonregional node).

T3N2aM1a: Tumor invades through the muscularis propria into the pericolorectal tissues; metastasis in 4-6 regional lymph nodes; metastasis confined to 1 organ or site (e.g. liver, lung, ovary, nonregional node).

T3N2bM1a: Tumor invades through the muscularis propria into the pericolorectal tissues; metastasis in 7 or more regional lymph nodes; metastasis confined to 1 organ or site (e.g. liver, lung, ovary, nonregional node).

T4aN0M1a: Tumor penetrates to the surface of the visceral peritoneum; no regional lymph node metastasis; metastasis confined to 1 organ or site (e.g. liver, lung, ovary, nonregional node).

T4aN1aM1a: Tumor penetrates to the surface of the visceral peritoneum; metastasis in 1 regional lymph node; metastasis confined to 1 organ or site (e.g. liver, lung, ovary, nonregional node).

IVB T3N0M1b: Tumor invades through the muscularis propria into the pericolorectal tissues; no regional lymph node metastasis; metastases in more than $1 \mathrm{organ} / \mathrm{site}$ or the peritoneum. 


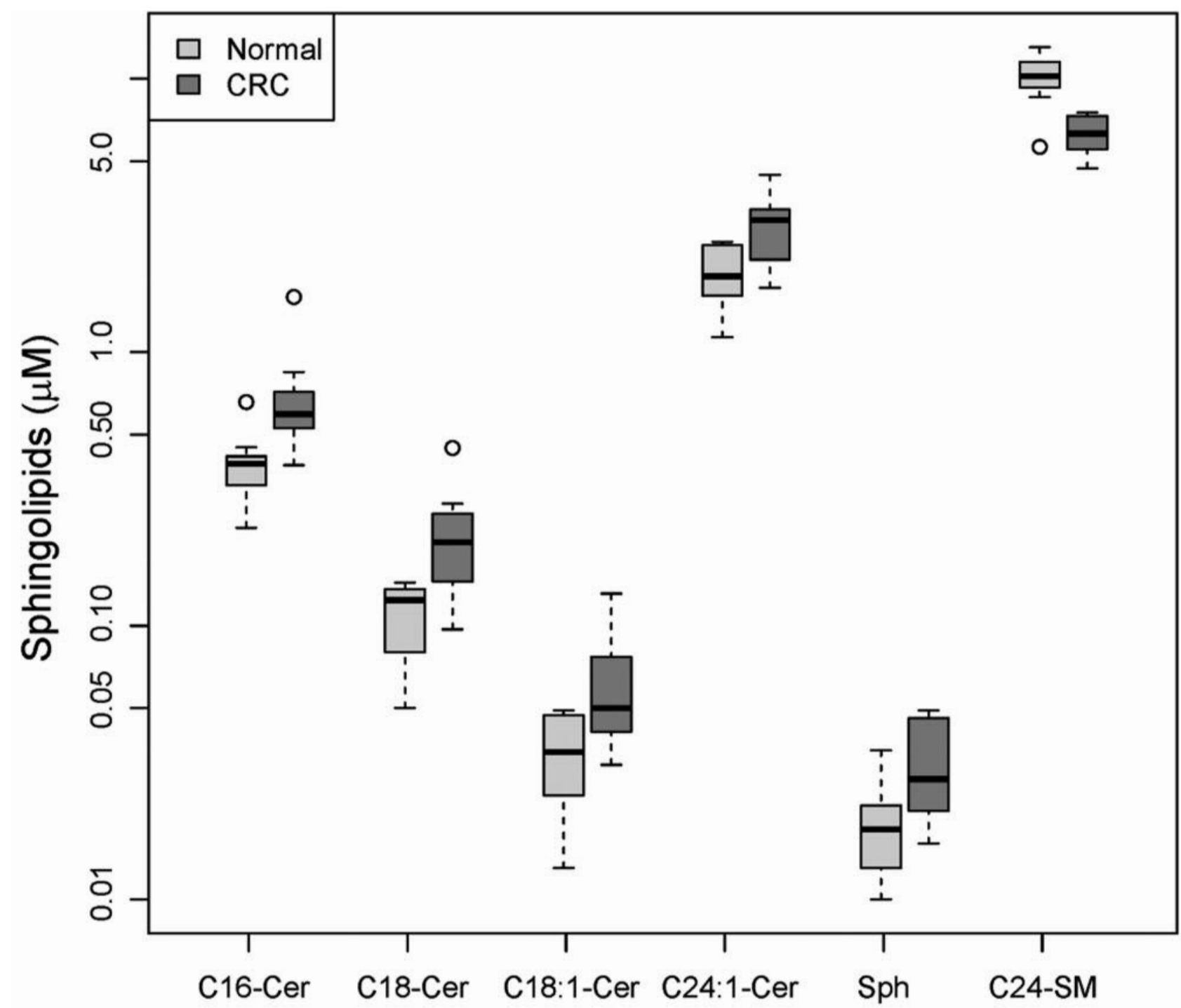

Figure 2. The serum levels of five sphingolipids were found to be significantly different in patients with stage IV CRC ( $n=10)$ compared to controls $(n=10)$. Data were log2-transformed (to improve normality) and modeled using a linear model. The significance of the group coefficient (CRC vs. control) was assessed via $t$-scores. $p$-Values were adjusted for multiple testing by using the false-discovery rate method to obtain $q$ values. A value of $q<0.05$ and fold change $>1.5$ were considered significant. All analyses were performed using the $R$ statistical environment. Sphingolipid concentrations are shown using boxes, with the horizontal thick line within each box representing the median value. The whiskers extend to the most extreme data point (minimum and maximum value), which is no more than 1.5-times the interquartile range (i.e. remaining $50 \%$ of the data) from each box. Cer, Ceramide; SM, sphingomyelin; Sph, sphingosine.

to an electrospray ionization source, and then analyzed by tandem mass spectrometry using a TSQ 7000 triple quadrupole mass spectrometer (Thermo-Fisher Scientific, Waltham, MA, USA) (7). The analyses included the full panel of sphingolipids, including ceramides, C16-dihydroceramide, sphingosine, sphingomyelins and hexosylceramides.

Statistical analysis. Differential concentration analysis: Sphingolipid concentrations were $\log _{2}$ transformed and compared between CRC and control groups using two-sample $t$-tests assuming equal variance. The difference in mean $\log$ concentration was converted into linear fold changes. $p$-Values were adjusted using the false-discovery rate method (8) to obtain $q$-values. Differential concentration was inferred based on values of $\mathrm{q}<0.05$ and fold change $>1.5$. In addition, a global test of association at the sphingolipid family level was conducted using the globaltest package in $\mathrm{R}$ statistical language environment (9). The null hypothesis for this test was that no sphingolipid in the family is associated with the outcome, i.e. with CRC vs. CRC-free. The three sphingolipid families tested were ceramides (12 individual ceramides), sphingomyelins (11 individual sphingomyelins), and hexosylceramides (nine individual hexosylceramides). 
Table II. The serum levels of six sphingolipids were found to be significantly changed in patients with stage IV colorectal cancer (CRC) $(n=10)$ compared to controls $(n=10)$. Data were log2-transformed (to improve normality) and modeled using a linear model. The significance of the group coefficient in the linear model (patients vs. controls) was assessed via t-scores. Two-tailed significance p-values were adjusted for multiple testing using the false-discovery rate method to obtain $q$ values. The magnitude of change in serum sphingolipid concentrations from patients with CRC compared to controls is reported as a fold change. A positive fold change indicates that the geometric average of serum concentrations of a sphingolipid from patients with CRC is higher compared to controls. A negative fold change indicates lower geometric average of serum concentrations of a sphingolipid from patients with CRC compared to controls. A value of $q<0.05$ and absolute fold change $>1.5$ were used to infer significant changes (shown in bold). All analyses were performed using the $R$ statistical environment.

\begin{tabular}{|c|c|c|c|}
\hline Sphingolipid & Fold change & $p$-Value & $q$-Value \\
\hline C16-Cer & 1.68 & 0.004 & 0.023 \\
\hline C18-Cer & 1.86 & 0.004 & 0.023 \\
\hline C18:1-Cer & 1.72 & 0.012 & 0.047 \\
\hline C24:1-Cer & 1.51 & 0.008 & 0.033 \\
\hline Sphingosine & 1.68 & 0.007 & 0.033 \\
\hline C24-SM & -1.59 & 0.000 & 0.001 \\
\hline C14-Cer & 1.47 & 0.087 & 0.183 \\
\hline C20-Cer & 1.32 & 0.174 & 0.283 \\
\hline C20:1-Cer & 1.42 & 0.102 & 0.205 \\
\hline C22-Cer & 1.23 & 0.078 & 0.179 \\
\hline C22:1-Cer & 1.20 & 0.080 & 0.179 \\
\hline C24-Cer & -1.01 & 0.931 & 0.982 \\
\hline C26-Cer & 1.16 & 0.474 & 0.621 \\
\hline C26:1-Cer & 1.57 & 0.061 & 0.179 \\
\hline C14-SM & 1.19 & 0.150 & 0.258 \\
\hline C16-SM & 1.14 & 0.074 & 0.179 \\
\hline C18-SM & -1.02 & 0.841 & 0.913 \\
\hline C18:1-SM & -1.19 & 0.108 & 0.206 \\
\hline C20-SM & -1.29 & 0.000 & 0.002 \\
\hline C20:1-SM & -1.32 & 0.001 & 0.011 \\
\hline C22-SM & -1.42 & 0.000 & 0.000 \\
\hline C22:1-SM & -1.36 & 0.000 & 0.002 \\
\hline C24:1-SM & 1.00 & 0.999 & 0.999 \\
\hline C26:1-SM & 1.69 & 0.053 & 0.179 \\
\hline Lyso-SM & 1.01 & 0.981 & 0.999 \\
\hline C16-HexCer & 1.36 & 0.129 & 0.234 \\
\hline C18-HexCer & 1.04 & 0.833 & 0.913 \\
\hline C20-HexCer & 1.38 & 0.194 & 0.283 \\
\hline C22-HexCer & -1.11 & 0.596 & 0.731 \\
\hline C22:1-HexCer & 1.52 & 0.064 & 0.179 \\
\hline C24-HexCer & -1.08 & 0.768 & 0.884 \\
\hline C24:1-HexCer & 1.44 & 0.072 & 0.179 \\
\hline C26-HexCer & -1.60 & 0.300 & 0.423 \\
\hline C26:1-HexCer & 1.34 & 0.346 & 0.469 \\
\hline $\mathrm{dhSph}$ & -1.05 & 0.591 & 0.731 \\
\hline DHS1P & 1.04 & 0.684 & 0.812 \\
\hline DHC16Cer & 1.45 & 0.183 & 0.283 \\
\hline Sph1P & 1.11 & 0.192 & 0.283 \\
\hline
\end{tabular}

Cer, Ceramide; HexCer, hexosylceramide; dhSph, dihydrosphingosine; DHS1P, dihydrosphingosine-1-phosphate; DHC16Cer, dihydroC16ceramide; SM, sphingomyelin; Sph1P, sphingosine-1-phosphate.
Correlation analysis: The correlation of concentrations of pairs of individual serum sphingolipids from patients and controls was assessed using Pearson correlation. Pairs of sphingolipids were considered significantly correlated provided that the adjusted $p$ values were less than 0.05 and the absolute correlation coefficient (r) was greater than 0.50. A hierarchical clustering of the matrix of correlation values with a Euclidean distance was performed using the heatmap function in $\mathrm{R}$.

\section{Results and Discussion}

Table II shows the differential analysis of the concentrations of 38 sphingolipids in sera obtained from CRC patients and controls. Serum sphingolipid levels from controls are similar to published serum sphingolipid levels from normal donors (7). Serum levels of six sphingolipids were significantly altered in patients with stage IV CRC compared to controls. The levels of C16-, C18-, C18:1- and C24:1-ceramide, as well as those of sphingosine, were significantly higher, and the levels of C24-sphingomyelin were significantly lower compared to controls (Table II; Figure 2). Moreover, the global test of association for the three families of sphingolipids showed that ceramides $(p=0.01)$ and sphingomyelins $(p=0.00025)$ were significantly associated with CRC, but not hexosylceramides $(p=0.24)$. The data suggest that ceramides and sphingomyelins but not hexosylceramides were implicated in stage IV CRC.

The heatmap depicted in Figure 3 summarizes the results of correlation analysis for serum sphingolipids from patients with CRC and normal donors. Three prominent clusters consisting of ceramides, sphingomyelins, and hexosylceramides were identified as significantly positively correlated (Figure 3, details D1, D2, and D3, respectively). A cluster of ceramides that was negatively correlated with the cluster of sphingomyelins was also identified (D4 in Figure 3).

We found 100 pairs of sphingolipids that were significantly correlated in both CRC and control groups (not shown). Among these, C18- and C18:1-ceramide were highly positively correlated $(\mathrm{r}=0.93)$, as well as $\mathrm{C} 16$-ceramide and C18-ceramide ( $\mathrm{r}=0.90)$. DihydroC16-ceramide and C16ceramide were also positively correlated $(\mathrm{r}=0.86)$. Because dihydroC16-ceramide is a metabolite from the de novo biosynthesis sphingolipid pathway, the data suggest that this pathway modulates blood levels of sphingolipids. Because the levels of C18- and C16-ceramide were higher in the CRC group compared to the control group, the data suggest enhanced up-regulation of CerS1 and CerS6, respectively, in patients with CRC.

A significant negative correlation was found for C22:1sphingomyelin and $\mathrm{C} 22$ : 1 -ceramide $(\mathrm{r}=-0.51)$ in both $\mathrm{CRC}$ and control groups. However, there was no significant correlation for C24-ceramide and C24-sphingomyelin, which had significantly lower levels in serum in patients with CRC (Table II, Figure2). The data suggest that if the blood levels 


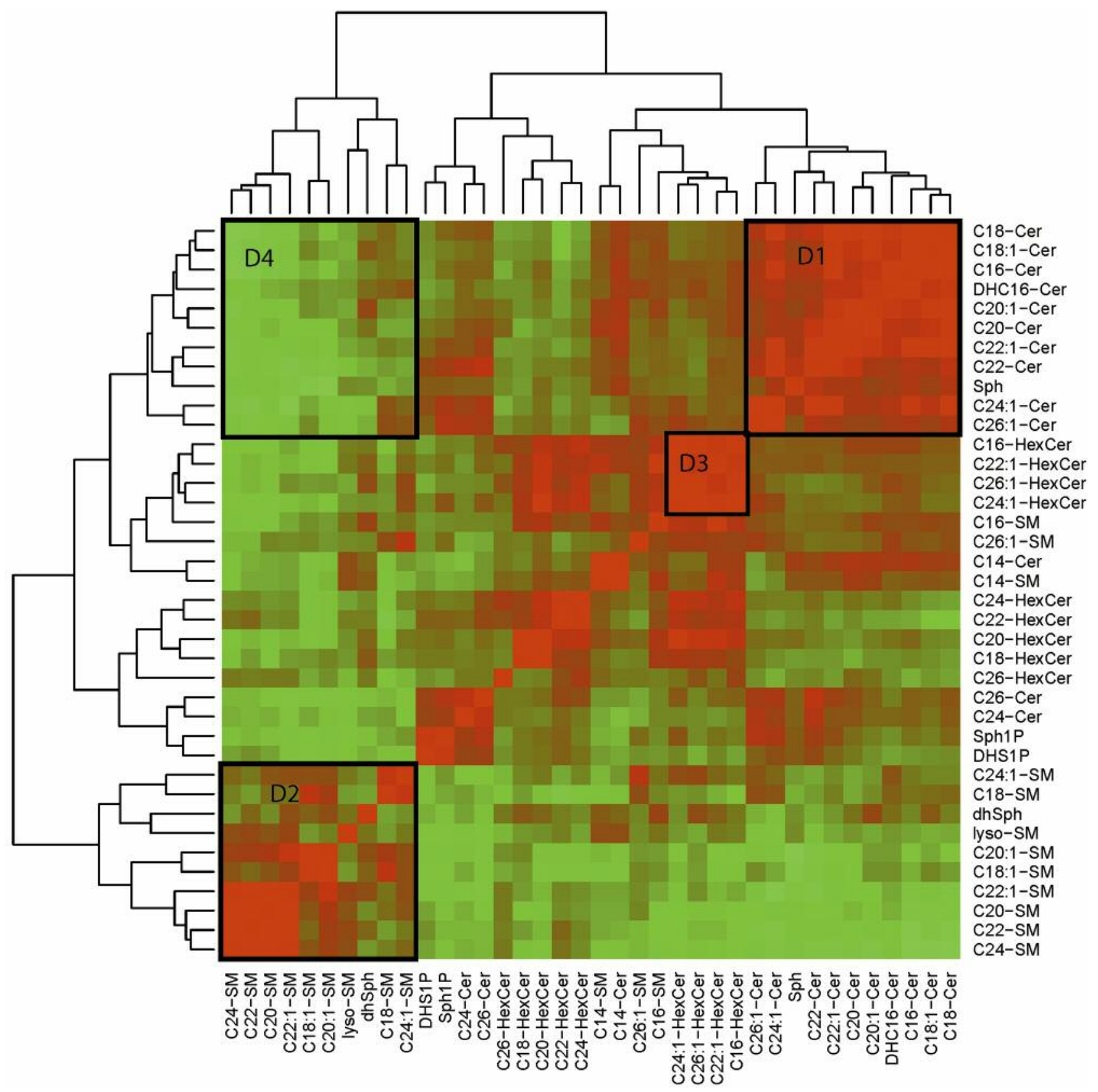

Figure 3. Sphingolipid heatmap. Using a color scale, the figure shows the Pearson correlation coefficient matrix computed for 20 serum samples obtained from 10 patients with CRC and 10 controls. The sphingolipid concentration data were log2-transformed. The correlation matrix was clustered using a hierarchical clustering algorithm with Euclidean distance. The branches of the tree drawn on top and on the left side of the figure define groups of sphingolipids with similar correlation coefficients. D1, D2 and D3 areas depict three prominent clusters of ceramides, sphingomyelins, and hexosylceramides, respectively, which were found to be significantly positively correlated. A cluster of ceramides that negatively correlated with the cluster of sphingomyelins is shown by D4. Cer, Ceramide; DHC16Cer, dihydroC16-ceramide; dhSph, dihydrosphingosine; dhSph1P, dihydrosphingosine-1-phosphate; HexCer, hexosylceramide; SM, sphingomyelin; Sph, sphingosine; Sph1P, sphingosine-1-phosphate.

of ceramide were affected by a sphingomyelinase, this was not a distinctive feature associated with stage IV CRC.

Sphingosine can be made from ceramide via ceramidase. If the blood levels of sphingosine were modulated by ceramidase, one would expect a negative correlation between sphingosine and ceramide. We observed, however, significant positive correlation for sphingosine and $\mathrm{C} 18$ ceramide, as well as for sphingosine and C16-ceramide 
( $\mathrm{r}=0.61$ for both pairs) in CRC patients and normal donors. The data from the correlation analysis do not support the role for ceramidase in sphingosine generation in CRC patients and normal donors or for the elevated sphingosine levels in CRC patients compared to controls. The data imply that a host response might have been reflected in observed significant changes in serum levels of sphingosine, as well as the four individual ceramides and C24-sphingomyelin in patients with CRC (cf. 10, 11).

One of the limitations of this study is that long-term outcomes of patients with CRC whose biosamples were used were not provided by Asterand. However, the immediate purpose of this novel preliminary study was to investigate the possibility that sphingolipids can serve as blood biomarkers indicative of stage IV CRC. Because we did observe significant alterations in the serum sphingolipid profile associated with stage IV CRC, a retrospective study using cohorts with known outcomes to validate the role of dysregulated sphingolipid metabolism as a predictor of mortality in patients with CRC is warranted.

Another limitation of the study is the small sample size. While we recorded significant results in spite of the small sample size, replication is needed in larger studies to confirm the findings. A study that includes patients with earlier stages of CRC is also warranted. In addition, mass spectrometry detected hexosylceramides and did not isolate glucosylceramides from galactosylceramides. These two lipids are isomers and require more time-consuming techniques to separate. As this was the first pilot study of these serum lipids in patients with CRC, these more extensive techniques were not conducted. We hypothesize that hexosylceramides reflect the levels of glucosylceramides, and not galactosylceramides. Future studies will need to verify this hypothesis by quantifying serum levels of each sphingolipid.

In conclusion, the present novel data suggest that sphingolipid metabolism is perturbed in the blood of patients with stage IV CRC, and that sphingolipids have the potential for serving as novel, non-invasive, inexpensive, and effective blood-based biomarkers to complement CRC staging for better prognoses and more personalized medicine.

\section{Acknowledgements}

This work was supported by the Faculty Research Award Program Individual Investigator Award from the Eugene Applebaum College of Pharmacy, Wayne State University (to DS). The mass spectrometry-related work at the Lipidomics Shared Resource Facility (Medical University of South Carolina) was supported by the National Cancer Institute Grants IPO1CA097132 and P30 CA 138313, NIH/NCRR SC COBRE Grant P20 RR017677, C06 RR018823 from the Extramural Research Facilities Program of the National Center for Research Resources.

\section{References}

1 Ludwig JA and Weinstein JN: Biomarkers in cancer staging, prognosis and treatment selection. Nat Rev Cancer 5: 845-856, 2005.

2 Kurek K, Lukaszuk B, Piotrowska DM, Wiesiolek P, Chabowska $\mathrm{AM}$ and Zendzian-Piotrowska M: Metabolism, physiological role, and clinical implications of sphingolipids in gastrointestinal tract. Biomed Res Int 2013: 908907, 2013.

3 Hertervig E, Nilsson A, Nyberg L and Duan RD: Alkaline sphingomyelinase activity is decreased in human colorectal carcinoma. Cancer 79: 448-453, 1997.

4 Wang C, Liu JN, Xu L, Mu YL and Sun P: Expression and significance of glucosylceramide synthase in colorectal carcinoma tissues. Eur Rev Med Pharmacol Sci 18: 3632-3637, 2014.

5 Schiffmann S, Sandner J, Birod K, Wobst I, Angioni C, Ruckhaberle E, Kaufmann M, Ackermann H, Lotsch J, Schmidt $\mathrm{H}$, Geisslinger $\mathrm{G}$ and Grosch $\mathrm{S}$ : Ceramide synthases and ceramide levels are increased in breast cancer tissue. Carcinogenesis 30: 745-752, 2009.

6 Suzuki M, Cao K, Kato S, Komizu Y, Mizutani N, Tanaka K, Arima C, Tai MC, Yanagisawa K, Togawa N, Shiraishi T, Usami N, Taniguchi T, Fukui T, Yokoi K, Wakahara K, Hasegawa Y, Mizutani Y, Igarashi Y, Inokuchi J, Iwaki S, Fujii S, Satou A, Matsumoto Y, Ueoka R, Tamiya-Koizumi K, Murate T, Nakamura M, Kyogashima $M$ and Takahashi T: Targeting ceramide synthase 6-dependent metastasis-prone phenotype in lung cancer cells. J Clin Invest 126: 254-265, 2016.

7 Hammad SM, Pierce JS, Soodavar F, Smith KJ, Al Gadban MM, Rembiesa B, Klein RL, Hannun YA, Bielawski J and Bielawska A: Blood sphingolipidomics in healthy humans: impact of sample collection methodology. J Lipid Res 51: 3074-3087, 2010.

8 Benjamini $\mathrm{Y}$ and Hochberg Y: Controlling the false discovery rate: a practical and powerful approach to multiple testing. J Royal Stat Soc B 57: 289-300, 1995.

9 Goeman JJ, van de Geer SA, de Kort F and van Houwelingen HC: A global test for groups of genes: testing association with a clinical outcome. Bioinformatics 20: 93-99, 2004.

10 Hannun YA and Newcomb B: A new twist to the emerging functions of ceramides in cancer: novel role for platelet acid sphingomyelinase in cancer metastasis. EMBO Mol Med 7: 692694, 2015.

11 Don AS, Lim XY and Couttas TA: Re-configuration of sphingolipid metabolism by oncogenic transformation. Biomolecules 4: 315-353, 2014.
Received January 13, 2017

Revised February 24, 2017

Accepted February 28, 2017 